\title{
INDICADORES DE DETERMINAÇÃO DE CORTES DE CULTIVARES DE AVEIA FORRAGEIRA
}

\author{
Ana Cândida Primavesi*; Odo Primavesi, Artur Chinellato; Rodolfo Godoy \\ Embrapa Pecuária Sudeste, C.P. 339 - CEP: 13560-970 - São Carlos, SP. \\ *Autor correspondente <anacan@cppse.embrapa.br>
}

\begin{abstract}
RESUMO: A produção de matéria seca total (MS) aumenta em função da idade da planta, mas simultaneamente ocorre decréscimo progressivo do seu valor nutritivo. O presente trabalho teve a finalidade de determinar a combinação de épocas de primeiro corte com a de intervalos de cortes da rebrota que proporcionasse a maior produção de matéria seca de aveia com as melhores características nutritivas. O delineamento experimental foi em blocos casualizados, com três repetições. Em 1994, os tratamentos foram organizados em fatorial $3 \mathrm{x}$ 4 [três indicadores de primeiro corte: 60 dias depois da emergência, início de emborrachamento (IE) e 10\% plantas encanadas (PE = alongamento do colmo, com elevação do meristema apical); quatro indicadores de intervalos de cortes da rebrota: 28 dias, 56 dias, IE, 10\% PE]. Foram testados dois cultivares: São Carlos e UPF 3. Em 1995 e 1996, os tratamentos foram organizados em esquema fatorial 4 × 3 (4 intervalos de corte da rebrota: 28, 35, 42 e 56 dias; três cultivares: São Carlos, UPF 3 e IAPAR 61). Foram avaliados: produção de MS, teores de N, Ca, P, Mg, digestibilidade "in vitro" e fibra em detergente neutro. Houve diferenças na produção de matéria seca e nas características nutritivas entre cultivares, épocas de primeiro corte e intervalos de cortes da rebrota. O primeiro corte deve ser feito com 10\% PE, e os cortes da rebrota devem ser efetuados em intervalos de 28 a 35 dias.
\end{abstract}

Palavras-chave: Avena sativa, A. byzantina, A. strigosa, alimentação animal, determinação bromatológica

\section{INDICATORS FOR CUTTING FORAGE OAT CULTIVARS}

\begin{abstract}
Dry matter (DM) yield increases as plants age, but at the same time there is a decrease in forage quality. Management studies on oat cultivars were performed to determine which indicators for the first cutting time and regrowth intervals would lead to the highest dry matter yield with the highest nutritive value. In 1994, a randomized complete block experimental design, with three replications was used, in a $3 \times 4$ factorial arrangement (the three first cut indicators were: sixty days after emergence, beginning of panicle differentiation (PD), and $10 \%$ of plants with culm elongation and apical meristem elevation (SE), while the four regrowth cutting intervals were: 28 days, 56 days, PD and 10\% SE. Two oat forage cultivars were used, São Carlos and UPF 3. In 1995 and 1996, a 4 × 3 factorial arrangement (four regrowth intervals: 28, 35, 42 and 56 days and three cultivars, São Carlos, UPF 3 and IAPAR 61) also in a randomized block design with three replications. Forage DM yield, crude protein, $\mathrm{Ca}, \mathrm{P}, \mathrm{Mg}$ content, 'in vitro' DM digestibility and neutral detergent fiber were determined. There were statistical differences for DM yield and nutritive characteristics among cultivars, first cutting time and regrowth cutting interval. The first cut should be performed with $10 \%$ SE and regrowth cuttings should be performed using an interval of 28 to 35 days.

Key words: Avena sativa, A. byzantina, A. strigosa, animal feeding, bromatological determinations
\end{abstract}

\section{INTRODUÇÃO}

A aveia (Avena spp) é uma gramínea anual de estação fria que pode ser cultivada em diferentes regiões, como fonte de forragem de boa qualidade nas formas verde, fenada e ensilada. No Estado de São Paulo apresenta-se como uma das alternativas para o cultivo no inverno, em sistemas intensivos de produção de leite. Possibilita aliar o cultivo de forrageira de clima temperado no inverno seco, com o de uma gramínea de clima tropical, milho ou sorgo para silagem no verão chuvoso, permitindo a diversificação na exploração agropecuária. Herling et al. (1998) relatam que a maior dificuldade encontrada pelos pecuaristas na implantação de sistemas de cultivo de inverno optando pelo cultivo de aveia, é a falta de cultivares alternativos de aveia com características desejáveis.
A aveia, como ocorre com as demais gramíneas herbáceas, apresenta em sua fase de crescimento vegetativo alta proporção de folhas, baixo teor de fibras e altos teores de proteína e ao passar para o estádio reprodutivo, sofre alterações que reduzem sua qualidade (Vilela et al., 1978; Sá, 1995). Isto determina queda na digestibilidade e consumo da forragem pelos animais. Ao passar do estádio vegetativo para o estádio reprodutivo sofre alterações morfológicas como alongamento do caule, morte ou senescência das folhas basais, e mudanças na porção citoplasmática da célula com decréscimos nos teores de proteínas, lipídios, e carboidratos solúveis. Ademais, ocorre elevação da porcentagem dos constituintes da parede celular e sua lignificação. A produção de matéria seca total, em função do tempo ou idade da planta, aumenta segundo o modelo sigmoidal, mas simultâneamente ocorre um progressivo decréscimo 
do seu valor nutritivo, particularmente na digestibilidade de sua matéria orgânica.

O declínio da digestibilidade é o resultado de três acontecimentos: redução na proporção dos tecidos mais digestíveis, menor concentração dos constituintes mais digestíveis e maior teor dos constituintes fibrosos (Vilela et al., 1978).

O lançamento e a recomendação para o Estado de São Paulo de cultivares de aveia (Godoy \& Batista, 1990a; Godoy \& Batista, 1990b, Godoy et al., 1998), e a demanda pela otimização da produção de leite em sistemas intensivos, justificam os estudos de manejo que possibilitem maiores produções de matéria seca de forragem, com melhores características nutritivas, para estes novos genótipos.

O presente trabalho teve por objetivo determinar a combinação de indicadores de épocas de primeiro corte com a de intervalos de cortes da rebrota que proporcione maior produção de matéria seca de forragem de aveia com melhores características nutritivas, possibilitando oferecer mais uma alternativa de alimentação para animais em sistemas intensivos de produção de leite, no período seco do inverno.

\section{MATERIAL E MÉTODOS}

Os experimentos foram instalados na Embrapa Pecuária Sudeste, em São Carlos, SP, em Latossolo Vermelho Distrófico típico. O preparo do solo consistiu em uma aração e duas gradagens, corrigindo-se a acidez pela aplicação de $1 \mathrm{t} \mathrm{ha}{ }^{-1}$ de calcário, efetuada antes da instalação dos experimentos. Anualmente, antes do plantio, foram aplicados $300 \mathrm{~kg} \mathrm{ha}^{-1}$ da fórmula 4-30-16 (NPK) e $300 \mathrm{~kg} \mathrm{ha}^{-1}$ de superfosfato simples, incorporados ao solo com enxada rotativa.

O experimento foi semeado em 16/05/94, num delineamento experimental em blocos casualizados com três repetições, com os tratamentos: três indicadores de primeiro corte: 60 dias após a emergência das plantas, início de emborrachamento (IE), e 10\% plantas encanadas ( $\mathrm{PE}=$ alongamento do colmo, com elevação do meristema apical) e quatro indicadores de intervalos de cortes da rebrota: 28 dias, 56 dias, IE, 10\% PE. Os cultivares de aveia usados foram: São Carlos e UPF 3. Em 1995 e em 1996 os experimentos foram instalados em duas épocas de plantio (17/04/95 e 15/05/95, e três repetições; 15/04/ 96 e 15/05/96, com quatro repetições), e o delineamento experimental em blocos casualizados e com os tratamentos: quatro intervalos de cortes da rebrota: 28,35 , 42 e 56 dias; três cultivares: São Carlos, UPF 3 e IAPAR 61. O primeiro corte foi realizado com $10 \% \mathrm{PE}$.

As parcelas eram constituídas por cinco linhas de $6 \mathrm{~m}$ de comprimento espaçadas de $20 \mathrm{~cm}$, com área útil de $3 \mathrm{~m}^{2}$. A semeadura foi manual com 70 sementes viáveis por metro. As capinas foram manuais, e a irrigação por aspersão, com $25 \mathrm{~mm}$ de água, parcelados em duas vezes por semana. Foram aplicados $40 \mathrm{~kg} \mathrm{ha}^{-1}$ de $\mathrm{N}$ e 40 $\mathrm{kg} \mathrm{ha}^{-1}$ de $\mathrm{K}_{2} \mathrm{O}$, no início do perfilhamento (20 a 25 dias após a emergência), e as mesmas doses após o primeiro corte. Após os cortes de rebrota foram aplicados $80 \mathrm{~kg}$ $\mathrm{ha}^{-1}$ de $\mathrm{N}$ e $80 \mathrm{~kg} \mathrm{ha}^{-1}$ de $\mathrm{K}_{2} \mathrm{O}$ em todos os tratamentos, parcelados de acordo com o número de cortes previsto. Os cortes foram manuais, à altura de $5-7 \mathrm{~cm}$ do solo. Após a pesagem da matéria fresca da parcela, foi retirada uma amostra com $500 \mathrm{~g}$ que foi colocada para secar a $60^{\circ} \mathrm{C}$, em estufa com circulação forçada de ar, até peso constante, para determinação do teor de água e posterior cálculo do teor de matéria seca. Essa amostra foi moída em moínho tipo Wiley, com peneira de 20 mesh (Sarruge \& Haag, 1974).

$O$ nitrogênio $(N)$ foi determinado pelo método microkjeldahl (AOAC, 1995), e a proteína bruta (PB) multiplicando-se o teor de N pelo fator 6,25. Por digestão nitroperclórica, obteve-se o extrato onde foram determinados cálcio $(\mathrm{Ca})$ e magnésio $(\mathrm{Mg})$ por titulação com EDTA e fósforo (P) por colorimetria (Malavolta et al., 1989). Determinaram-se também a digestibilidade "in vitro" da matéria seca (DIVMS) pelo método de Tilley e Terry modificado (Silva, 1981) e fibra em detergente neutro (FDN) (Van Soest, 1963). As determinações bromatológicas foram corrigidas para matéria seca a $105^{\circ} \mathrm{C}$. Os dados obtidos foram analisados utilizando-se o procedimento GLM do programa SAS (1993).

\section{RESULTADOS E DISCUSSÃO}

Em 1994, os experimentos visaram determinar especialmente 0 indicador mais adequado para primeiro corte. A análise de variância (TABELA 1) encontrou diferenças entre indicadores de primeiro corte para matéria seca digestível e protéica, sendo que o contraste de médias destacou o indicador 10\% PE. Foi encontrada diferença entre cultivares para matéria seca total, digestível e protéica, com destaque para o cv. São Carlos. A produção de matéria seca protéica, porém, dependeu de todas as interações, necessitando análise específica para cada cultivar. Verificou-se diferença para intervalos de cortes de rebrota para matéria seca total e digestível, havendo, porém, interação com o primeiro corte.

As maiores produções de matéria seca digestível e protéica foram obtidas, no caso do cv. São Carlos, quando o primeiro corte foi efetuado com $10 \% \mathrm{PE}$ e os cortes de rebrota com intervalos de 28 dias e com o cv. UPF 3, quando o primeiro corte foi efetuado com $10 \%$ PE e os cortes de rebrota com intervalos de 56 dias (TABELA 3). Com o cv. UPF 3 verificou-se que, quando o primeiro corte foi efetuado com $10 \% \mathrm{PE}$, embora no corte da primeira rebrota com 56 dias as plantas estivessem florescidas, os valores de PB e DIVMS se mantiveram elevados e os de FDN baixos (TABELA 4).

Em 1994, o indicador estádio fisiológico para determinar o intervalo de cortes de rebrota não foi adequado para o cv. UPF 3, pois ocorreu redução do período de intervalo de cortes quando os cortes eram 
TABELA 1 - Análise de variância para as produções de matéria seca total, digestível e protéica.

\begin{tabular}{|c|c|c|c|c|c|c|c|c|}
\hline Causa de & $\mathrm{GL}$ & & $\mathrm{F}$ & $\mathrm{GL}$ & QM & $\mathrm{F}$ & QM & $\mathrm{F}$ \\
\hline Variação & \multicolumn{3}{|c|}{ matéria seca total } & \multicolumn{3}{|c|}{ matéria seca digestível } & \multicolumn{2}{|c|}{ matéria seca protéica } \\
\hline . & $-\cdots--$ & 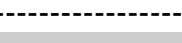 & 事 & 1994 & 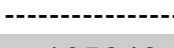 & $-\cdots-\cdot$ & 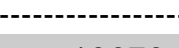 & $\ldots$ \\
\hline Bloco & 2 & $13195 \approx$ & & 2 & 165242 & & 13679 & \\
\hline IC & 2 & 962001 & 0,37 & 2 & 1582850 & $11,17^{\star *}$ & 381359 & $36,76^{\star *}$ \\
\hline $\mathbb{R}$ & 3 & 30505398 & $118,87^{* *}$ & 2 & 4468404 & $31,52^{* *}$ & 2782 & 0,27 \\
\hline$I C^{*} \mathbb{R}$ & 6 & 668027 & $2,60^{*}$ & 2 & 1184604 & $8,36^{* *}$ & 133811 & $12,90^{* *}$ \\
\hline res (a) & 22 & 25662 c & & 12 & $14175 C$ & & 10374 & \\
\hline Var & 1 & 61321392 & $186,84^{\star *}$ & 1 & 7457391 & $28,54^{\star *}$ & 152051 & $14,47^{\star *}$ \\
\hline $\operatorname{Var}^{*} \mathrm{IC}$ & 2 & 34403 & 0,10 & 2 & 727171 & 2,78 & 195863 & $18,63^{* *}$ \\
\hline $\operatorname{Var}^{*} \mathbb{R}$ & 3 & 381619 & 1,16 & 1 & 128809 & 0,49 & 223929 & $21,30^{* *}$ \\
\hline $\operatorname{Var}^{*} \mid C^{*} \mathbb{R}$ & 6 & $107553 \varepsilon$ & $3,26^{*}$ & 2 & 2905798 & $11,12^{\star *}$ & 456298 & $43,41^{\text {** }}$ \\
\hline res (b) & 24 & 7876711 & & 12 & 261274 & & 10511 & \\
\hline Total & 71 & & & 38 & & & & \\
\hline CV (\%) & & 10,1 & & & 10,4 & & 8,8 & \\
\hline DMS IC & & & 303,28 & & & 323,54 & & 87,53 \\
\hline DMS $\mathbb{R}$ & & & 405,19 & & & 446,53 & & 120,80 \\
\hline DMS Var & & & 278,70 & & & 357,73 & & 71,75 \\
\hline Bloco & 2 & 1487743 & & 2 & & & 38187 & \\
\hline Var & 2 & 11831496 & $48,18^{\star *}$ & 2 & 894137 & $92,34^{* *}$ & 155580 & $14,31^{\star *}$ \\
\hline $\mathbb{R}$ & 3 & 7365787 & $30,00^{* *}$ & 3 & 11687768 & $5,09^{* *}$ & 185818 & $17,09^{* *}$ \\
\hline $\operatorname{Var}^{*} \mathbb{R}$ & 6 & 505225 & 2,06 & 6 & 644358 & $2,67^{*}$ & 7367 & 0,68 \\
\hline res (a) & 22 & $24556 ?$ & & 22 & 338284 & & 10871 & \\
\hline EP & 1 & 3057830 & $7,09^{*}$ & 1 & 126580 & $14,19^{\star *}$ & 5228 & 0,49 \\
\hline$E P^{*}$ Var & 2 & 660866 & 1,53 & 2 & 3027849 & 1,69 & 11699 & 1,09 \\
\hline$E P^{*} \mathbb{R}$ & 3 & 3070143 & $7,12^{* *}$ & 3 & 360974 & 2,64 & 20765 & 1,93 \\
\hline$E P^{*} \operatorname{Var}^{*} \mathbb{R}$ & 6 & 500723 & 1,16 & 6 & $56418 C$ & 0,56 & 7610 & 0,71 \\
\hline res (b) & 24 & 431438 & & 24 & 119611 & & 10749 & \\
\hline Total & 71 & & & 71 & & & & \\
\hline CV (\%) & & 9,1 & & & 8,6 & & 9,0 & \\
\hline DMS Var & & & 296,67 & & & $213,0 \mathrm{C}$ & & 62,42 \\
\hline DMS $\mathbb{R}$ & & & 396,36 & & & 284,57 & & 83,40 \\
\hline DMS EP & & & 319,53 & & & 224,68 & & 50,44 \\
\hline Bloco & 3 & 1941526 & & 3 & $89314 \varepsilon$ & & 46175 & \\
\hline Var & 2 & 2955908 & $7,09^{\star \star}$ & 2 & 3871866 & $19,50^{\star \star}$ & 137297 & $12,57^{\star *}$ \\
\hline $\mathbb{R}$ & 3 & 24929532 & $59,83^{\star \star}$ & 3 & 2948609 & $14,85^{\star \star}$ & 185927 & $17,02^{\star *}$ \\
\hline $\operatorname{Var}^{*} \mathbb{R}$ & 6 & 1019448 & $2,45^{\star}$ & 6 & 266399 & 1,34 & 20504 & 1,88 \\
\hline res (a) & 33 & 416644 & & 33 & 198585 & & 10921 & \\
\hline EP & 1 & 4229297 & $14,89^{\star *}$ & 1 & 2558719 & $16,40^{\star \star}$ & 1138 & 0,09 \\
\hline$E P^{*}$ Var & 2 & $208129 C$ & $7,33^{* *}$ & 2 & 785970 & $5,04^{*}$ & 136954 & $10,87^{\star *}$ \\
\hline$E P^{*} \mathbb{R}$ & 3 & 520426 & 1,83 & 3 & 370280 & 2,37 & 25958 & 2,06 \\
\hline$E P^{*} \operatorname{Var}^{*} \mathbb{R}$ & 6 & $31044 C$ & 1,09 & 6 & 223042 & 1,43 & 19036 & 1,51 \\
\hline res (b) & 36 & 284000 & & 36 & 156016 & & 12603 & \\
\hline Total & 95 & & & 95 & & & & \\
\hline CV (\%) & & 6,5 & & & 6,3 & & 8,1 & \\
\hline DMS Var & & & 328,31 & & & 226,66 & & 53,15 \\
\hline DMS $\mathbb{R}$ & & & 436,54 & & & 301,38 & & 70,68 \\
\hline DMS EP & & & 220,62 & & & 163,52 & & 46,48 \\
\hline
\end{tabular}

Obs: Var = cultivar, $\mathrm{IC}=$ indicador do primeiro corte, $\mathrm{IR}=$ intervalo de corte da rebrota, $\mathrm{EP}=$ época de plantio. DMS $=$ diferença mínima significativa (Tukey a $5 \%$ ), CV = coeficiente de variação. 
TABELA 2 - Análise de variância para os teores de PB, FDN e DIVMS.

\begin{tabular}{|c|c|c|c|c|c|c|c|}
\hline Causa de & $\mathrm{GL}$ & QM & $\mathrm{F}$ & QM & $\mathrm{F}$ & QM & $\mathrm{F}$ \\
\hline Variação & \multicolumn{5}{|c|}{----------- PB ----------- ---------- FDN --------- } & \multicolumn{2}{|c|}{------- DIVMS ------ } \\
\hline Bloco & 2 & 5,696 & & 0,123 & & 1,615 & \\
\hline Var & 2 & 8,752 & $12,08^{* *}$ & 21,206 & $22,72^{* *}$ & 227,959 & $161,56^{* *}$ \\
\hline $\mathbb{R}$ & 3 & 22,076 & $40,57^{* *}$ & 106,608 & $114,23^{* *}$ & 190,640 & $110,81^{* *}$ \\
\hline $\operatorname{Var}^{*} \mathbb{R}$ & 6 & 1,809 & 3,32 ** & 10,316 & $11,05^{* *}$ & 4,389 & 2,56 * \\
\hline res (a) & 22 & 0,544 & & 0,933 & & 1,720 & \\
\hline EP & 1 & 46,820 & $58,03^{\text {** }}$ & 3,387 & 3,44 & 5,059 & 2,11 \\
\hline$E P^{*} \operatorname{Var}$ & 2 & 3,818 & $4,73^{*}$ & 4,003 & $4,06^{*}$ & 9,440 & 3,93 * \\
\hline$E P^{*} \mathbb{R}$ & 3 & 6,736 & $8,35^{* *}$ & 5,190 & 5,26 ** & 28,459 & $11,86^{* *}$ \\
\hline$E P^{*} \operatorname{Var}^{*} \mathbb{R}$ & 6 & 0,943 & 1,17 & 1,570 & 1,59 & 3,786 & 1,58 \\
\hline res (b) & 24 & 0,807 & & 0,986 & & 2,400 & \\
\hline Total & 71 & & & & & & \\
\hline CV (\%) & & 5,1 & & 1,9 & & 2,0 & \\
\hline DMS Var & & & 0,442 & & 0,578 & & 0,785 \\
\hline DMS $\mathbb{R}$ & & & 0,590 & & 0,773 & & 1,049 \\
\hline DMS EP & & & 0,437 & & 0,483 & & 0,754 \\
\hline Bloco & 3 & 1,062 & & 0,009 & & 3,586 & \\
\hline Var & 2 & 7,898 & $14,55^{* *}$ & 11,811 & $8,47^{\text {** }}$ & 108,620 & $43,97^{\text {** }}$ \\
\hline $\mathbb{R}$ & 3 & 10,202 & $92,46^{* *}$ & 213,892 & $153,45^{* *}$ & 253,213 & 102,50 ** \\
\hline $\operatorname{Var}^{\star} \mathbb{R}$ & 6 & 0,969 & 1,78 & 6,473 & $4,64^{\text {** }}$ & 5,065 & 2,05 \\
\hline res (a) & 33 & 0,543 & & 1,394 & & 2,470 & \\
\hline EP & 1 & 19,004 & $28,76^{* *}$ & 21,635 & $13,27^{* *}$ & 1,762 & 0,92 \\
\hline$E P^{*} \operatorname{Var}$ & 2 & 1,722 & 2,61 & 8,535 & $5,24^{* *}$ & 12,387 & 6,50 ** \\
\hline$E P^{*} \mathbb{R}$ & 3 & 1,845 & 2,79 & 9,467 & $5,81^{\text {** }}$ & 13,112 & $6,88^{* *}$ \\
\hline$E P^{*} \operatorname{Var}^{*} \mid R$ & 6 & 0,519 & 0,79 & 2,518 & 1,55 & 4,518 & $2,37^{*}$ \\
\hline res (b) & 36 & 0,661 & & & & 1,906 & \\
\hline Total & 95 & & & & & & \\
\hline CV (\%) & & 4,5 & & 2,4 & & 1,8 & \\
\hline DMS Var & & & 0,375 & & 0,601 & & 0,799 \\
\hline DMS $\mathbb{R}$ & & & 0,498 & & 0,798 & & 1,063 \\
\hline DMS EP & & & 0,336 & & 0,529 & & 0,572 \\
\hline
\end{tabular}

Obs: Var = cultivar, IC = indicador do primeiro corte, IR = intervalo de corte da rebrota, EP = época de plantio. DMS = diferença mínima significativa (Tukey a 5\%), CV = coeficiente de variação. 
TABELA 3 - Produção (primeiro corte + rebrotas) de matéria seca total, matéria seca digestível e de proteína bruta dos cultivares de aveia São Carlos e UPF 3, 1994.

\begin{tabular}{|c|c|c|c|c|c|}
\hline \multicolumn{2}{|c|}{ Tratamentos } & \multirow{2}{*}{$\begin{array}{c}\text { No total } \\
\text { de Cortes }\end{array}$} & \multicolumn{3}{|c|}{ Produção de matéria seca } \\
\hline \multirow[t]{2}{*}{$1^{\circ}$ Corte } & \multirow[t]{2}{*}{ Cortes de Rebrota } & & Total & Digestível & Protéica \\
\hline & & & --.-.. & $-\mathrm{kg} \mathrm{ha}^{-1}$ & ------ \\
\hline
\end{tabular}

\begin{tabular}{|c|c|c|c|c|c|}
\hline \multicolumn{6}{|c|}{ cv. São Carlos } \\
\hline 60 dias & 28 dias & 5 & $7059 \mathrm{~b}$ & 5553 a & $1365 a b$ \\
\hline 60 dias & 56 dias & 3 & $8202 a$ & $5904 a$ & 1192 bc \\
\hline 60 dias & $\mathbb{E}$ & 5 & $5698 \mathrm{c}$ & - & - \\
\hline 60 dias & $10 \% \mathrm{PE}$ & 6 & $5564 \mathrm{c}$ & - & - \\
\hline $\mathbb{E}$ & 28 dias & 5 & $7001 \mathrm{~b}$ & - & - \\
\hline $\mathbb{E}$ & 56 dias & 3 & $7880 a b$ & 5676 a & $1133 \mathrm{c}$ \\
\hline $\mathbb{E}$ & $\mathbb{E}$ & 5 & $5967 \mathrm{c}$ & - & - \\
\hline $\mathbb{E}$ & $10 \% \mathrm{PE}$ & 6 & $5247 \mathrm{c}$ & - & - \\
\hline $10 \%$ PE & 28 dias & 6 & $7192 a b$ & 5660 a & 1469 a \\
\hline $10 \% \mathrm{PE}$ & 56 dias & 3 & $7941 \mathrm{ab}$ & $5619 a$ & $1090 \mathrm{c}$ \\
\hline $10 \% \mathrm{PE}$ & $\mathbb{E}$ & 5 & $5946 \mathrm{c}$ & - & - \\
\hline $10 \% \mathrm{PE}$ & $10 \% \mathrm{PE}$ & 7 & $5247 c$ & $4314 \mathrm{~b}$ & $1331 a b$ \\
\hline
\end{tabular}

cV. UPF 3

\begin{tabular}{lccccc}
60 dias & 28 dias & 5 & $5383 \mathrm{~cd}$ & $4376 \mathrm{bc}$ & $1018 \mathrm{~b}$ \\
60 dias & 56 dias & 3 & $5621 \mathrm{bc}$ & $4134 \mathrm{bc}$ & $801 \mathrm{c}$ \\
60 dias & $\mathbb{E}$ & 7 & $4387 \mathrm{ef}$ & - & - \\
\hline 60 dias & $10 \% \mathrm{PE}$ & 7 & $3540 \mathrm{~g}$ & - & - \\
$\mathbb{E}$ & 28 dias & 5 & $5053 \mathrm{~cd}$ & $4046 \mathrm{bc}$ & $988 \mathrm{~b}$ \\
$\mathbb{E}$ & 56 dias & 3 & $6304 \mathrm{~b}$ & $4530 \mathrm{~b}$ & $864 \mathrm{bc}$ \\
$\mathbb{E}$ & $\mathbb{E}$ & 7 & $3806 \mathrm{fg}$ & - & - \\
$\mathbb{E}$ & $10 \% \mathrm{PE}$ & 7 & $3412 \mathrm{~g}$ & - & - \\
$10 \% \mathrm{PE}$ & 28 dias & 6 & $4725 \mathrm{de}$ & $3717 \mathrm{c}$ & $1003 \mathrm{~b}$ \\
$10 \% \mathrm{PE}$ & 56 dias & 3 & $7843 \mathrm{a}$ & $6162 \mathrm{a}$ & $1834 \mathrm{a}$ \\
$10 \% \mathrm{PE}$ & $\mathbb{E}$ & 7 & $3324 \mathrm{~g}$ & - & - \\
$10 \% \mathrm{PE}$ & $10 \% \mathrm{PE}$ & 7 & $3399 \mathrm{~g}$ & - & - \\
\hline
\end{tabular}

Valores na coluna seguidos da mesma letra não diferem $(P>0,05$, teste $t)$. MS digestível= MS total $x$ DIVMS/100. IE = início emborrachamento. $\mathrm{PE}=$ plantas encanadas.

efetuados com $10 \%$ PE, e cortes efetuados no IE. O mesmo ocorreu com o cv. São Carlos, porém com menor redução do período de intervalo de cortes de rebrota. Muldoon (1986) explica que o estádio de desenvolvimento da planta vai se tornando mais avançado nas rebrotas sucessivas, o que ocorreu neste experimento, tornando os indicadores IE e 10\% PE, inadequados.
Em 1995 e 1996, encontraram-se diferenças entre cultivares e intervalos de cortes de rebrota e épocas de plantio, para as produções de matéria seca total, digestível e protéica (TABELA 1), e para teores de $\mathrm{PB}$, de FDN e DIVMS (TABELA 2).

Em 1995, para a produção de matéria seca total, digestiva e protéica, houve destaque para o cv. São Carlos 
TABELA 4 - Teor de proteína bruta (PB), digestibilidade "in vitro" (DIVMS) e fibra em detergente neutro (FDN), por corte, do cV. UPF 3, média de três repetições, 1994.

\begin{tabular}{|c|c|c|c|c|c|c|c|}
\hline \multicolumn{2}{|c|}{ Tratamento } & \multirow[t]{2}{*}{$1^{\circ} \mathrm{C}$} & \multirow[t]{2}{*}{$1^{\mathrm{a}} \mathrm{R}$} & \multirow[t]{2}{*}{$2^{\mathrm{a}} \mathrm{R}$} & \multirow[t]{2}{*}{$3^{a} \mathrm{R}$} & \multirow[t]{2}{*}{$4^{\mathrm{a}} \mathrm{R}$} & \multirow[t]{2}{*}{$5^{\mathrm{a}} \mathrm{R}$} \\
\hline $1^{\circ} \mathrm{C}$ & IC (dias) & & & & & & \\
\hline \multicolumn{8}{|c|}{ PB (\%) } \\
\hline 60 dias & 28 & 16,3 & 27,1 & 22,6 & 19,8 & 15,4 & - \\
\hline 60 dias & 56 & 16,3 & 12,7 & 14,2 & - & - & - \\
\hline $\mathbb{E}$ & 28 & 15,9 & 22,6 & 18,2 & 20,3 & 16,6 & - \\
\hline $\mathbb{E}$ & 56 & 19,5 & 11,1 & 14,1 & - & - & - \\
\hline $10 \%$ PE. & 28 & 25,5 & 19,8 & 24,8 & 20,1 & 14,5 & 17,7 \\
\hline $10 \%$ PE. & 56 & 25,2 & 25,3 & 13,2 & - & - & - \\
\hline \multicolumn{8}{|c|}{ DIVMS (\%) } \\
\hline 60 dias & 28 & 83,8 & 84,0 & 78,0 & 78,4 & 70,6 & - \\
\hline 60 dias & 56 & 83,8 & 66,7 & 60,5 & - & - & - \\
\hline $\mathbb{E}$ & 28 & 86,1 & 80,6 & 76,1 & 76,7 & 68,8 & - \\
\hline $\mathbb{E}$ & 56 & 86,1 & 66,1 & 63,4 & - & - & - \\
\hline $10 \%$ PE. & 28 & 85,5 & 77,6 & 82,1 & 78,6 & 71,4 & 71,0 \\
\hline $10 \%$ PE. & 56 & 85,5 & 82,5 & 54,2 & - & - & - \\
\hline \multicolumn{8}{|c|}{ FDN (\%) } \\
\hline 60 dias & 28 & 49,6 & 42,2 & 52,4 & 51,1 & 62,7 & - \\
\hline 60 dias & 56 & 49,6 & 62,9 & 62,6 & - & - & - \\
\hline $\mathbb{E}$ & 28 & 44,3 & 43,9 & 54,3 & 53,8 & 61,8 & - \\
\hline $\mathbb{E}$ & 56 & 44,3 & 65,1 & 61,9 & - & - & - \\
\hline $10 \%$ PE. & 28 & 44,1 & 51,1 & 46,6 & 53,9 & 62,4 & 58,1 \\
\hline $10 \% \mathrm{PE}$ & 56 & 44,1 & 46,4 & 61,0 & - & - & - \\
\hline
\end{tabular}

$\mathrm{C}=$ corte; $\mathrm{IE}$ = início de emborrachamento; $\mathrm{PB}=$ proteína bruta; $\mathrm{R}=$ rebrota; $\mathrm{PE}=$ plantas encanadas; $\mathrm{IC}=$ intervalo de cortes.

seguido do cv. IAPAR 61 e em 1996 o cv. IAPAR 61 superou em matéria seca protéica. Em 1995 e 1996, para os intervalos de cortes de rebrota houve destaque para 56 dias considerando matéria seca total e digestível, e 28 a 35 dias para matéria seca protéica. Em 1995 e 1996, houve diferenças entre épocas de plantio somente para matéria seca total e digestível, com vantagem para abril, dependendo do cultivar no ano de 1996.

Para os cultivares IAPAR 61 e UPF 3 , os teores de $\mathrm{PB}$ foram iguais nos dois anos e maiores que os teores do cv. São Carlos. Os teores de FDN foram iguais e menores para o cv. São Carlos, respectivamente em 1995 e 1996, em relação ao cv. IAPAR 61, sendo que o cv.UPF 3 apresentou os maiores teores. Para os teores de DIVMS, em ambos os anos, o cv. IAPAR 61 foi superior aos cvs. São Carlos e UPF 3 , sendo que o cv.UPF 3 apresentou os menores teores. Estes dados indicam melhores características nutritivas da forragem para os dois cvs. de aveias forrageiras: São Carlos (aveia amarela) e IAPAR 61 (aveia preta), em relação ao cv. UPF 3 (aveia duplo propósito: forragem e grãos).

Nos dois anos, os maiores teores de PB e DIVMS e os menores teores de FDN foram obtidos com os intervalos de cortes de 28 dias, seguidos pelos intervalos de 35,42 e 56 dias. Isto indica que as melhores características nutritivas da forragem de aveia são obtidas com intervalos de cortes de rebrota de 28 a 35 dias. Houve diferença entre épocas de plantio para teores de PB, em ambos os anos, e para teores de FDN em 1996, com teores maiores para o plantio em maio.

Em 1995 (TABELA 5), as maiores produções de matéria seca digestível e protéica na primeira época de plantio foram obtidas para os cultivares São Carlos e IAPAR 61, com intervalos de corte de 28 e de 35 dias, e para o cultivar UPF 3 de 35 dias. Na segunda época de 
TABELA 5 - Produção $\left(1^{\circ}\right.$ corte + cortes de rebrota) de matéria seca total e protéica e teores de PB, FDN e DIVMS, dos cvs. São Carlos, UPF 3 e IAPAR 61, em duas épocas de plantio, 1995.

\begin{tabular}{|c|c|c|c|c|c|c|c|c|}
\hline \multirow{2}{*}{$\begin{array}{l}\text { Época de } \\
\text { plantio }\end{array}$} & \multirow{2}{*}{$\begin{array}{c}\text { Tratamentos } \\
\text { IC }\end{array}$} & \multirow[t]{2}{*}{ NTC } & \multicolumn{3}{|c|}{ Produção de matéria seca } & \multirow[t]{2}{*}{ PB } & \multirow[t]{2}{*}{ FDN } & \multirow[t]{2}{*}{ DIVMS } \\
\hline & & & Total & Digestível & Protéica & & & \\
\hline & dias & & \multicolumn{3}{|c|}{ - } & \multicolumn{3}{|c|}{ 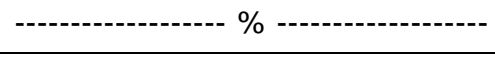 } \\
\hline \multicolumn{9}{|c|}{ cv. São Carlos } \\
\hline $17 / 04$ & 28 & 6 & $7520 \mathrm{~b}$ & $6068 a b$ & $1410 \mathrm{a}$ & $18,3 \mathrm{a}$ & $47,6 \mathrm{c}$ & 81,0 a \\
\hline $17 / 04$ & 35 & 5 & 8681 a & 6736 a & $1277 a b$ & $15,3 \mathrm{~b}$ & $49,6 \mathrm{~b}$ & 77,8 b \\
\hline $17 / 04$ & 42 & 4 & $7725 \mathrm{~b}$ & $5845 \mathrm{~b}$ & $1127 \mathrm{~b}$ & $16,7 \mathrm{~b}$ & $50,1 \mathrm{~b}$ & $76,5 \mathrm{~b}$ \\
\hline $17 / 04$ & 56 & 3 & $8253 a b$ & $6179 a b$ & $1141 \mathrm{~b}$ & $16,1 \mathrm{~b}$ & 53,3 a & $76,3 \mathrm{~b}$ \\
\hline $15 / 05$ & 28 & 5 & $7065 \mathrm{~b}$ & $5687 \mathrm{~b}$ & 1331 a & $19,1 \mathrm{a}$ & $48,8 \mathrm{c}$ & $80,1 \mathrm{a}$ \\
\hline $15 / 05$ & 35 & 4 & $7414 \mathrm{~b}$ & $5965 a b$ & $1158 a b$ & $17,9 \mathrm{~b}$ & $48,5 \mathrm{c}$ & 81,4 a \\
\hline $15 / 05$ & 42 & 4 & $7321 \mathrm{~b}$ & $5477 \mathrm{~b}$ & $1076 \mathrm{~b}$ & $16,8 \mathrm{bc}$ & $52,6 \mathrm{~b}$ & 76,7 b \\
\hline $15 / 05$ & 56 & 3 & $9890 \mathrm{a}$ & 6488 a & $1163 a b$ & $15,7 \mathrm{c}$ & 56,3 a & $72,3 \mathrm{c}$ \\
\hline \multicolumn{9}{|c|}{ cV. UPF 3} \\
\hline $17 / 04$ & 28 & 6 & $6112 \mathrm{~b}$ & $4774 \mathrm{~b}$ & $1162 \mathrm{a}$ & $18,9 a$ & $50,4 \mathrm{~b}$ & 77,9 a \\
\hline $17 / 04$ & 35 & 5 & 7726 a & $5629 a$ & $1195 a$ & $16,0 \mathrm{~b}$ & $52,8 \mathrm{a}$ & 73,6 b \\
\hline $17 / 04$ & 42 & 4 & $6954 a b$ & $4903 \mathrm{~b}$ & $1043 a b$ & $16,7 \mathrm{~b}$ & 53,5 a & 72,7 b \\
\hline $17 / 04$ & 56 & 3 & 7453 a & $4717 \mathrm{~b}$ & $906 \mathrm{~b}$ & $16,4 \mathrm{~b}$ & 53,7 a & $71,0 \mathrm{c}$ \\
\hline $15 / 05$ & 28 & 5 & $5830 \mathrm{~b}$ & 4545 & 1193 a & $20,6 a$ & $50,8 \mathrm{~b}$ & $77,0 \mathrm{a}$ \\
\hline $15 / 05$ & 35 & 4 & $5587 \mathrm{~b}$ & 4291 & $1039 a b$ & $19,5 \mathrm{a}$ & $51,5 \mathrm{~b}$ & $76,2 \mathrm{a}$ \\
\hline $15 / 05$ & 42 & 4 & $6401 a b$ & 4070 & $1007 \mathrm{~b}$ & $18,0 \mathrm{~b}$ & 55,3 a & $68,5 \mathrm{~b}$ \\
\hline $15 / 05$ & 56 & 3 & $7331 \mathrm{a}$ & 4353 & $969 \mathrm{~b}$ & $16,8 \mathrm{c}$ & $52,7 \mathrm{~b}$ & $65,8 \mathrm{~b}$ \\
\hline \multicolumn{9}{|c|}{ cv. IAPAR 61} \\
\hline $17 / 04$ & 28 & 6 & $6938 a b$ & 5645 & $1264 \mathrm{a}$ & $17,1 a b$ & $48,5 \mathrm{c}$ & $81,3 \mathrm{a}$ \\
\hline $17 / 04$ & 35 & 5 & $6852 \mathrm{~b}$ & 5444 & $1270 \mathrm{a}$ & $17,6 \mathrm{a}$ & $49,2 \mathrm{c}$ & $80,3 \mathrm{ab}$ \\
\hline $17 / 04$ & 42 & 4 & $6662 \mathrm{~b}$ & 5096 & $1067 \mathrm{~b}$ & $16,1 \mathrm{~b}$ & $52,1 \mathrm{~b}$ & 79,0 b \\
\hline $17 / 04$ & 56 & 3 & 7786 a & 5662 & $1124 a b$ & $17,0 a b$ & $55,0 \mathrm{a}$ & $75,8 \mathrm{c}$ \\
\hline $15 / 05$ & 28 & 5 & $6285 \mathrm{~b}$ & $5291 \mathrm{ab}$ & $1360 \mathrm{a}$ & $20,6 a$ & $48,4 \mathrm{C}$ & $82,5 \mathrm{a}$ \\
\hline $15 / 05$ & 35 & 4 & $5870 \mathrm{~b}$ & $4869 \mathrm{~b}$ & $1189 a b$ & $20,5 \mathrm{a}$ & $48,5 \mathrm{c}$ & $82,3 \mathrm{a}$ \\
\hline $15 / 05$ & 42 & 4 & $6812 \mathrm{~b}$ & $5161 \mathrm{ab}$ & $1208 a b$ & $19,1 \mathrm{~b}$ & $52,6 \mathrm{~b}$ & 78,4 b \\
\hline $15 / 05$ & 56 & 3 & 7912 a & $5579 a$ & $1089 \mathrm{~b}$ & $17,0 \mathrm{c}$ & $55,1 \mathrm{a}$ & $75,4 \mathrm{c}$ \\
\hline
\end{tabular}

Valores na coluna seguidos da mesma letra não diferem $(P>0,05$, teste t). MS digestível=MS total $\times$ DIVMS/100; NTC $=$ número total de cortes; IC = intervalo de cortes.

plantio, para o cv. São Carlos, com intervalos de corte de 35 dias; cv. UPF 3 de 28 e 35 dias, e cv. IAPAR 61 com intervalos de cortes da rebrota de 28 dias.

Em 1995 (TABELA 6), com o cultivar UPF 3, não se repetiu o que foi verificado em 1994 nas plantas que foram submetidas a intervalos de corte de 56 dias, pois também com as plantas florescidas, os teores de PB e DIVMS não se mantiveram elevados e nem os de FDN baixos, o que é esperado, mas não sendo possível explicar o ocorrido em 1994.

Em 1996, considerando-se as produções de matéria seca digestível e protéica e os valores de DIVMS 
TABELA 6 - Teor de proteína bruta, fibra detergente neutro e digestibilidade in vitro por corte e por época de plantio do cv. UPF 3, 1995.

\begin{tabular}{|c|c|c|c|c|c|c|c|c|}
\hline $\begin{array}{l}\text { Época de } \\
\text { Plantio }\end{array}$ & $\begin{array}{c}\text { Tratamento IC } \\
\text { (dias) }\end{array}$ & $1^{\circ} \mathrm{C}$ & $1^{a} \mathrm{R}$ & $2^{a} \mathrm{R}$ & $3^{a} \mathrm{R}$ & $4^{a} \mathrm{R}$ & $5^{\mathrm{a}} \mathrm{R}$ & M \\
\hline \multicolumn{9}{|c|}{ PB (\%) } \\
\hline $17 / 04$ & 28 & 22,7 & 19,3 & 22,9 & 20,4 & 16,2 & 12,1 & 18,9 \\
\hline $17 / 04$ & 35 & 22,7 & 15,3 & 17,7 & 14,9 & 9,3 & - & 16,0 \\
\hline $17 / 04$ & 42 & 22,7 & 14,1 & 18,9 & 11,3 & - & - & 16,7 \\
\hline $17 / 04$ & 56 & 22,7 & 9,5 & 16,9 & - & - & - & 16,4 \\
\hline $15 / 05$ & 28 & 26,4 & 20,6 & 21,6 & 19,1 & 15,0 & - & 20,6 \\
\hline $15 / 05$ & 35 & 26,4 & 16,1 & 21,4 & 14,0 & - & - & 19,5 \\
\hline $15 / 05$ & 42 & 26,4 & 12,6 & 21,2 & 12,0 & - & - & 18,0 \\
\hline $15 / 05$ & 56 & 26,4 & 9,6 & 14,2 & - & - & - & 16,8 \\
\hline \multicolumn{9}{|c|}{ FDN (\%) } \\
\hline $17 / 04$ & 28 & 41,0 & 52,8 & 44,8 & 48,5 & 54,3 & 61,1 & 50,4 \\
\hline $17 / 04$ & 35 & 41,0 & 60,4 & 48,5 & 50,2 & 63,9 & - & 52,8 \\
\hline $17 / 04$ & 42 & 41,0 & 62,0 & 51,7 & 59,4 & - & - & 53,5 \\
\hline $17 / 04$ & 56 & 41,0 & 64,4 & 55,8 & - & - & - & 53,7 \\
\hline $15 / 05$ & 28 & 40,7 & 51,7 & 48,2 & 53,4 & 60,3 & - & 50,8 \\
\hline $15 / 05$ & 35 & 40,7 & 53,4 & 56,2 & 55,8 & - & - & 51,5 \\
\hline $15 / 05$ & 42 & 40,7 & 65,2 & 53,6 & 61,7 & - & - & 55,3 \\
\hline $15 / 05$ & 56 & 40,7 & 62,2 & 55,3 & - & - & - & 52,7 \\
\hline \multicolumn{9}{|c|}{ DIVMS (\%) } \\
\hline $17 / 04$ & 28 & 82,4 & 77,7 & 85,3 & 77,0 & 81,3 & 74,9 & 77,9 \\
\hline $17 / 04$ & 35 & 82,4 & 72,5 & 76,8 & 73,1 & 72,3 & - & 73,6 \\
\hline $17 / 04$ & 42 & 82,4 & 69,3 & 73,8 & 65,5 & - & - & 72,7 \\
\hline $17 / 04$ & 56 & 82,4 & 58,0 & 72,5 & - & - & - & 71,0 \\
\hline $15 / 05$ & 28 & 81,2 & 85,0 & 74,9 & 77,9 & 71,6 & - & 77,0 \\
\hline $15 / 05$ & 35 & 81,2 & 80,0 & 73,5 & 70,0 & - & - & 76,2 \\
\hline $15 / 05$ & 42 & 81,2 & 57,4 & 76,2 & 59,2 & - & - & 68,5 \\
\hline $15 / 05$ & 56 & 81,2 & 53,1 & 63,2 & - & - & - & 65,8 \\
\hline
\end{tabular}

$\mathrm{C}=$ corte, $\mathrm{R}=$ rebrota, $\mathrm{IC}=$ intervalo de cortes.

(TABELA 7), os melhores indicadores de cortes da rebrota para características nutritivas da forragem foram com os cvs. São Carlos e IAPAR 61, para as duas épocas, intervalos de cortes de 28 dias; com o cv. UPF 3, na primeira época, intervalos de cortes de 35 dias e na segunda época, de 28 dias.

Não ocorreu a mesma resposta aos tratamentos nos dois anos consecutivos, provavelmente devido às condições climáticas, mas ficou evidente que, para se obter boas produções de matéria seca de aveia aliadas às características nutritivas, os cortes da rebrota para os três cultivares estudados devem ser efetuados entre os intervalos de 28 a 35 dias.

De maneira geral, os teores de $\mathrm{Ca}, \mathrm{P}$ e Mg (TABELA 7) foram maiores na segunda época de plantio para os três cultivares. As exigências em minerais dos animais são afetadas por espécie animal, raça, intensidade de produção, condições ambientais, idade etc. (Souza, 1985). 
TABELA 7 - Produção ( $1^{\circ}$ corte + cortes de rebrota) de matéria seca total e protéica e teores de PB, FDN e DIVMS, dos cvs. São Carlos, UPF 3 e IAPAR 61, em duas épocas de plantio, 1996

\begin{tabular}{|c|c|c|c|c|c|c|c|c|c|c|}
\hline Época & Tratamentos & Produ & ào de matér & ia seca & PB & FDN & DIVMS & $\mathrm{Ca}$ & $\mathrm{P}$ & $\mathrm{Mg}$ \\
\hline plantio & (IC) & Total & Digestível & Protéica & & & & & & \\
\hline
\end{tabular}

cv. São Carlos

\begin{tabular}{lllllllllll}
$15 / 04$ & 28 & $7431 \mathrm{~b}$ & $6030 \mathrm{~b}$ & $1422 \mathrm{a}$ & $18,4 \mathrm{a}$ & $48,8 \mathrm{~d}$ & $80,8 \mathrm{a}$ & $0,29 \mathrm{~b}$ & $0,28 \mathrm{a}$ & 0,11 \\
$15 / 04$ & 35 & $8080 \mathrm{~b}$ & $6154 \mathrm{~b}$ & $1410 \mathrm{a}$ & $17,5 \mathrm{ab}$ & $50,4 \mathrm{c}$ & $77,0 \mathrm{~b}$ & $0,35 \mathrm{a}$ & $0,25 \mathrm{ab}$ & 0,11 \\
$15 / 04$ & 42 & $8365 \mathrm{~b}$ & $6236 \mathrm{~b}$ & $1263 \mathrm{~b}$ & $16,5 \mathrm{bc}$ & $52,6 \mathrm{~b}$ & $76,7 \mathrm{~b}$ & $0,31 \mathrm{~b}$ & $0,23 \mathrm{~b}$ & 0,11 \\
$15 / 04$ & 56 & $10028 \mathrm{a}$ & $7253 \mathrm{a}$ & $1216 \mathrm{~b}$ & $15,0 \mathrm{c}$ & $54,9 \mathrm{a}$ & $74,8 \mathrm{~b}$ & $0,30 \mathrm{~b}$ & $0,22 \mathrm{~b}$ & 0,10 \\
$15 / 05$ & 28 & $7684 \mathrm{c}$ & $6245 \mathrm{bc}$ & $1536 \mathrm{a}$ & $19,8 \mathrm{a}$ & $48,3 \mathrm{c}$ & $81,0 \mathrm{a}$ & 0,40 & 0,25 & 0,13 \\
$15 / 05$ & 35 & $7444 \mathrm{c}$ & $5897 \mathrm{c}$ & $1360 \mathrm{a}$ & $18,9 \mathrm{a}$ & $50,3 \mathrm{c}$ & $78,9 \mathrm{~b}$ & 0,36 & 0,29 & 0,13 \\
$15 / 05$ & 42 & $8855 \mathrm{~b}$ & $6772 \mathrm{ab}$ & $1547 \mathrm{a}$ & $17,6 \mathrm{~b}$ & $52,8 \mathrm{~b}$ & $74,2 \mathrm{c}$ & 0,35 & 0,30 & 0,12 \\
$15 / 05$ & 56 & $10310 \mathrm{a}$ & $6878 \mathrm{a}$ & $1405 \mathrm{a}$ & $16,6 \mathrm{~b}$ & $57,3 \mathrm{a}$ & $69,7 \mathrm{~d}$ & 0,43 & 0,26 & 0,12 \\
\hline
\end{tabular}

cv. UPF 3

$\begin{array}{lllllllllll}15 / 04 & 28 & 6987 \mathrm{~b} & 5503 \mathrm{c} & 1405 \mathrm{ab} & 19,5 \mathrm{a} & 50,2 \mathrm{~b} & 78,3 \mathrm{a} & 0,39 \mathrm{a} & 0,29 \mathrm{a} & 0,09 \mathrm{~b} \\ 15 / 04 & 35 & 8208 \mathrm{~b} & 6325 \mathrm{ab} & 1497 \mathrm{a} & 18,8 \mathrm{ab} & 53,2 \mathrm{a} & 77,5 \mathrm{a} & 0,33 \mathrm{ab} & 0,24 \mathrm{ab} & 0,12 \mathrm{ab} \\ 15 / 04 & 42 & 8084 \mathrm{~b} & 5870 \mathrm{bc} & 1315 \mathrm{~b} & 17,8 \mathrm{bc} & 53,4 \mathrm{a} & 74,3 \mathrm{~b} & 0,35 \mathrm{ab} & 0,22 \mathrm{~b} & 0,17 \mathrm{a} \\ 15 / 04 & 56 & 10275 \mathrm{a} & 6859 \mathrm{a} & 1329 \mathrm{~b} & 16,5 \mathrm{c} & 55,1 \mathrm{a} & 70,6 \mathrm{c} & 0,29 \mathrm{~b} & 0,21 \mathrm{~b} & 0,09 \mathrm{~b} \\ 15 / 05 & 28 & 6810 \mathrm{~b} & 5515 \mathrm{~b} & 1431 \mathrm{a} & 20,9 \mathrm{a} & 49,2 \mathrm{c} & 80,0 \mathrm{a} & 0,46 & 0,39 \mathrm{a} & 0,12 \\ 15 / 05 & 35 & 6780 \mathrm{~b} & 5331 \mathrm{~b} & 1301 \mathrm{ab} & 19,8 \mathrm{~b} & 52,5 \mathrm{~b} & 78,3 \mathrm{a} & 0,45 & 0,31 \mathrm{~b} & 0,10 \\ 15 / 05 & 42 & 7190 \mathrm{~b} & 5459 \mathrm{~b} & 1132 \mathrm{c} & 16,9 \mathrm{c} & 56,1 \mathrm{a} & 75,0 \mathrm{~b} & 0,37 & 0,27 \mathrm{bc} & 0,09 \\ 15 / 05 & 56 & 9083 \mathrm{a} & 6014 \mathrm{a} & 1175 \mathrm{bc} & 16,9 \mathrm{c} & 55,1 \mathrm{a} & 71,7 \mathrm{c} & 0,40 & 0,25 \mathrm{c} & 0,10\end{array}$

CV. IAPAR 61

\begin{tabular}{lllllllllll}
$15 / 04$ & 28 & $7942 \mathrm{~b}$ & 6457 & $1599 \mathrm{a}$ & $19,5 \mathrm{a}$ & $48,9 \mathrm{c}$ & $81,4 \mathrm{a}$ & $0,51 \mathrm{a}$ & 0,27 & 0,12 \\
$15 / 04$ & 35 & $8086 \mathrm{~b}$ & 6516 & $1480 \mathrm{ab}$ & $17,9 \mathrm{~b}$ & $50,6 \mathrm{bc}$ & $80,8 \mathrm{ab}$ & $0,50 \mathrm{ab}$ & 0,26 & 0,13 \\
$15 / 04$ & 42 & $8838 \mathrm{a}$ & 6867 & $1412 \mathrm{ab}$ & $17,7 \mathrm{~b}$ & $51,6 \mathrm{~b}$ & $78,9 \mathrm{bc}$ & $0,47 \mathrm{bc}$ & 0,25 & 0,12 \\
$15 / 04$ & 56 & $9294 \mathrm{a}$ & 7009 & $1299 \mathrm{~b}$ & $16,9 \mathrm{~b}$ & $55,8 \mathrm{a}$ & $77,0 \mathrm{c}$ & $0,46 \mathrm{c}$ & 0,23 & 0,12 \\
$15 / 05$ & 28 & $7376 \mathrm{c}$ & 6049 & 1593 & 21,1 & 49,4 & 81,7 & 0,52 & 0,25 & 0,17 \\
$15 / 05$ & 35 & $7623 \mathrm{bc}$ & 6235 & 1458 & 18,9 & 51,8 & 81,7 & 0,54 & 0,27 & 0,17 \\
$15 / 05$ & 42 & $8333 \mathrm{ab}$ & - & - & - & - & - & 0,52 & 0,26 & 0,16 \\
$15 / 05$ & 56 & $9091 \mathrm{a}$ & 6538 & 1323 & 17,2 & 58,2 & 74,4 & 0,52 & 0,23 & 0,16 \\
\hline
\end{tabular}

Valores na coluna seguidos da mesma letra não diferem entre si $(P>0,05$, teste $t)$. MS digestível=MS total $x$ DIVMS/100; IC = intervalo de cortes.

Conhecendo-se a necessidade de minerais de vacas leiteiras com pesos de 500 a $600 \mathrm{~kg}$ e produções de leite variando de 17 a 20 litros/ dia, ou seja, 0,51\% de $\mathrm{Ca}, 0,20 \%$ de $\mathrm{Mg}$, e $0,33 \%$ de $\mathrm{P}$, e $15 \%$ de PB (NRC, 1988), verifica-se que apenas a forragem do cv. IAPAR 61 apresentou teores de $\mathrm{Ca}$ compatíveis com os requerimentos deste nutriente, para esta categoria animal, nas duas épocas de plantio. Para os três cultivares, os teores de $\mathrm{P}$ e $\mathrm{Mg}$ na forragem foram menores que as exigências destes animais, devendo, portanto serem complementados na ração, exceto para o cv. UPF 3, que, na segunda época de plantio e para o 
TABELA 8 - Teor de PB, FDN e DIVMS, por corte, por época de plantio, no ano de 1996, para o cv. São Carlos.

\begin{tabular}{|c|c|c|c|c|c|c|c|c|}
\hline Época plantio & IC (dias) & $1^{\circ} \mathrm{C}$ & $1^{\mathrm{a}} \mathrm{R}$ & $2^{\mathrm{a}} \mathrm{R}$ & $3^{a} \mathrm{R}$ & $4^{\mathrm{a}} \mathrm{R}$ & $5^{\mathrm{a}} \mathrm{R}$ & Média \\
\hline \multicolumn{9}{|c|}{ PB (\%) } \\
\hline $15 / 04$ & 28 & 25,5 & 19,6 & 20,9 & 14,2 & 16,8 & 13,2 & 18,4 \\
\hline $15 / 04$ & 35 & 25,5 & 17,1 & 19,0 & 13,1 & 13,1 & - & 17,5 \\
\hline $15 / 04$ & 42 & 25,5 & 12,7 & 15,9 & 12,0 & - & - & 16,5 \\
\hline $15 / 04$ & 56 & 25,5 & 9,1 & 10,7 & - & - & - & 15,0 \\
\hline $15 / 05$ & 28 & 27,1 & 22,0 & 18,0 & 14,8 & 17,3 & - & 19,8 \\
\hline $15 / 05$ & 35 & 27,1 & 16,4 & 17,8 & 14,5 & - & - & 18,9 \\
\hline $15 / 05$ & 42 & 27,1 & 15,6 & 14,6 & 13,1 & - & - & 17,6 \\
\hline $15 / 05$ & 56 & 27,1 & 11,6 & 11,2 & - & - & - & 16,6 \\
\hline \multicolumn{9}{|c|}{ FDN (\%) } \\
\hline $15 / 04$ & 28 & 42,4 & 49,9 & 45,7 & 50,4 & 50,9 & 53,8 & 48,8 \\
\hline $15 / 04$ & 35 & 42,4 & 54,4 & 44,2 & 55,5 & 54,9 & - & 50,3 \\
\hline $15 / 04$ & 42 & 42,4 & 58,1 & 48,6 & 60,6 & - & - & 52,4 \\
\hline $15 / 04$ & 56 & 42,4 & 59,3 & 62,2 & - & - & - & 54,6 \\
\hline $15 / 05$ & 28 & 43,8 & 48,7 & 47,1 & 51,3 & 50,7 & - & 48,3 \\
\hline $15 / 05$ & 35 & 43,8 & 50,4 & 51,3 & 55,7 & - & - & 50,3 \\
\hline $15 / 05$ & 42 & 43,8 & 51,3 & 54,6 & 61,3 & - & - & 52,8 \\
\hline $15 / 05$ & 56 & 43,8 & 63,9 & 64,0 & - & - & - & 57,3 \\
\hline \multicolumn{9}{|c|}{ DIVMS (\%) } \\
\hline $15 / 04$ & 28 & 84,4 & 80,4 & 83,9 & 78,4 & 81,0 & 77,0 & 80,9 \\
\hline $15 / 04$ & 35 & 84,4 & 71,3 & 79,0 & 74,8 & 75,1 & - & 76,9 \\
\hline $15 / 04$ & 42 & 84,4 & 72,4 & 80,5 & 68,9 & - & - & 76,5 \\
\hline $15 / 04$ & 56 & 84,4 & 72,0 & 67,4 & - & - & - & 74,6 \\
\hline $15 / 05$ & 28 & 82,5 & 82,8 & 82,9 & 78,1 & 78,5 & - & 81,0 \\
\hline $15 / 05$ & 35 & 82,5 & 80,9 & 77,7 & 74,4 & - & - & 78,9 \\
\hline $15 / 05$ & 42 & 82,5 & 77,0 & 73,8 & 63,5 & - & - & 74,2 \\
\hline $15 / 05$ & 56 & 82,5 & 65,9 & 60,8 & - & - & - & 69,7 \\
\hline
\end{tabular}

$\mathrm{C}=$ corte, $\mathrm{R}=$ rebrota, $\mathrm{IC}=$ intervalo de cortes.

intervalo de cortes de 28 dias, mostrou teores de $\mathrm{P}$ adequados a esta categoria animal. Os teores de PB da forragem dos três cultivares, nas duas épocas de plantio, estão acima do exigido por estes animais.

Para o cv. São Carlos (TABELA 8), no manejo com intervalos de cortes de rebrota de 28 e de 35 dias, embora os teores de FDN tenham aumentado com os sucessivos cortes, e os de DIVMS tenham diminuído, o que também foi verificado por Edmisten et al. (1998), ainda são suficientemente altos para não limitar o consumo. Segundo
Hibbs \& Conrad (1975), a quantidade de alimento que uma vaca pode ingerir em um período de 24 horas, é limitado pelo resíduo indigestível no trato digestivo, quando a digestibilidade está abaixo de $67 \%$. Esses autores também relatam que quando a digestibilidade está acima de $67 \%$, outros fatores limitam o consumo voluntário, mas nas vacas com capacidade genética para alta produção de leite, o consumo de energia digestível é o fator mais limitante. e que com a forragem madura, a digestibilidade, o consumo voluntário de matéria seca e a produção de leite diminuem. 


\section{CONCLUSÕES}

Recomenda-se no Estado de São Paulo, para os cvs. de aveia São Carlos, UPF 3 e IAPAR 61, o sistema de cortes para produção de forragem, mantendo as características nutritivas da mesma: o primeiro corte efetuado quando as plantas de aveia iniciam o estádio de elongação do colmo (primeiro nó visível), que nas nossas condições ocorre entre 38 e 45 dias após a emergência, e cortes da rebrota com intervalos de 28 dias (4 e 5 cortes) a 35 dias ( 3 e 4 cortes, quando a semeadura for feita em abril e maio, respectivamente), independente do ano.

Nesse manejo de cortes a forragem dos cvs. São Carlos, UPF 3 e IAPAR 61 apresenta alto valor nutritivo constituindo, portanto, uma alternativa para alimentação de animais em sistemas intensivos de produção de leite, no período seco do inverno.

\section{REFERÊNCIAS BIBLIOGRÁFICAS}

ASSOCIATION OF OFFICIAL ANALYTICAL CHEMISTS. Official methods of analysis of AOAC International. 16.ed. Arlington: AOAC, 1995.v.1.

EDMISTEN, K.L.; GREEN, J.T; MUELLER JR., J.P.; BURNS, J.C. Winter annual small grain forage potential: II. Quantification of nutritive characteristics of four small grain species at six growth stages. Communication Soil Science Plant Analysis, v.29, p. 881-899, 1998.

GODOY, R. ; BATISTA, L.A.R. Recomendação de cultivares de aveia forrageira para a região de São Carlos, SP. São Carlos: Embrapa Pecuária Sudeste, 1990a. 6p. (Comunicado Técnico, 3).

GODOY, R. ; BATISTA, L.A.R. Avaliação de germoplasma de aveia forrageira em São Carlos, SP. Revista Sociedade Brasileira de Zootecnia, v.19, p.235-242, 1990b.

GODOY, R.; PRIMAVESI, A.C.; BATISTA, L.A.R; CESAR, F.C; REIS, R.A.; HERLING,V.;YAMANAKA, R.N.; DANTAS, R.; SILVA, J.R. Recomendação de cultivares de aveia para o Estado de São Paulo. São Carlos: Embrapa Pecuária Sudeste, 1998. 9p. (Comunicado Técnico, 19).
HERLING, V.R.; SILVA, J.R.; GODOY, R.; PRIMAVESI, A.C.; IUZ, P.H.C.; VAN MELIS, M.H.; CARRE, C.R.; GOMIDE, C.A.; LIMA, C.G. Estudos de alguns parâmetros agronômicos de cultivares de aveia (Avena spp.). In: REUNIÃO DA SOCIEDADE BRASILEIRA DE ZOOTECNIA, 35., Botucatu, 1998. Anais. Botucatu: SBZ, 1998. p.524-526.

HIBBS, J.B.; CONRAD, H.R. Minimum concentrate feeding for efficient milk production. World Animal Review, v.15, p.33-38, 1975.

MALAVOLTA, E., VITTI, G.C., OLIVEIRA, S. A. Avaliação do estado nutricional das plantas: princípios e aplicações. Piracicaba: Associação Brasileira para a Pesquisa de Potassa e Fosfato, 1989. 201p.

MULDOON, D.K. Dry matter accumulation and changes in forage quality during primary growth and three regrowths of irrigated winter cereals. Australian Journal of Experimental Agriculture, v.26, p.87-98, 1986.

NATIONAL RESEARCH COUNCIL. Subcommittee on Dairy Cattle Nutrition. Nutrient requeriments of dairy cattle. 6.ed. Washington: National Academy Press, 1988. 157p.

SÁ, J.P.G. Utilização da aveia na alimentação animal. Londrina: IAPAR, 1995. 20p. (Circular, 87).

SARRUGE, J.R; HAAG, H.P. Análises químicas em plantas. Piracicaba: ESALQ/USP, 1974. 56p.

SAS INSTITUTE. SAS/STAT user's guide: statistics, versão 6 . 4.ed. Cary: Statistical Analysis System Institute, 1993. v.2, $1686 p$.

SILVA, D.J. Análise de alimentos: métodos químicos e biológicos. Viçosa: Imprensa Universitária da UFV, 1981. $166 \mathrm{p}$.

SOUZA, J.C. Formulação de misturas minerais para bovinos. In: SIMPÓSIO SOBRE NUTRIÇÃO DE BOVINOS, 3. Piracicaba, 1985. Anais. Piracicaba: FEALQ. 1985. p.87-98.

VAN SOEST, P.J. Use of detergents in the analysis of fibrous feed: II. A rapid method for the determination of fiber and lignin. Journal of the Association of Official Agricultural Chemists, v.46, p.829-835, 1963.

VILELA, H.; GOMIDE, J.A.; SILVA, J.F.C. Valor nutritivo da aveia forrageira (Avena byzantina L.) sob as formas de verde, silagem e feno. Revista da Sociedade Brasileira de Zootecnia v.7, p.145-157, 1978.

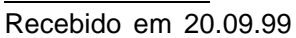

